\title{
THÉODORE TAPSOBA
}

\section{Automates calculant la complexité de suites automatiques}

\author{
Journal de Théorie des Nombres de Bordeaux, tome 6, no 1 (1994), \\ p. $127-134$ \\ <http://www.numdam.org/item?id=JTNB_1994_6_1_127_0>
}

(C) Université Bordeaux 1, 1994, tous droits réservés.

L'accès aux archives de la revue « Journal de Théorie des Nombres de Bordeaux » (http://jtnb.cedram.org/) implique l'accord avec les conditions générales d'utilisation (http://www.numdam.org/conditions). Toute utilisation commerciale ou impression systématique est constitutive d'une infraction pénale. Toute copie ou impression de ce fichier doit contenir la présente mention de copyright.

\section{Numdam}

Article numérisé dans le cadre du programme

Numérisation de documents anciens mathématiques

http://www.numdam.org/ 
Journal de Théorie des Nombres

de Bordeaux 6 (1994), 127-134

\title{
Automates calculant la complexité de suites automatiques
}

\author{
par THÉODORE TAPSOBA
}

\begin{abstract}
RÉSUME - Le point fixe $u$ d'une substitution injective uniforme de module $\sigma$ sur un alphabet $A$ est examiné du point de vue du nombre $P(u, n)$ de ses blocs distincts de longueur $n$. Lorsque $u$ est minimal et $A$ de cardinal deux, nous construisons un automate pour la suite $n \rightarrow P(u, n+1)-P(u, n)$.

ABSTRACT - A fixed point $u$ of an injective substitution of constant length $\sigma$ on an alphabet $A$ is considered in relation with the number $P(u, n)$ of its distinct $n$-blocks. When $u$ is minimal and $A$ a set of two elements, we prove that the sequence $n \rightarrow P(u, n+1)-P(u, n)$ is obtained by an automaton which is built explicitly.
\end{abstract}

\section{Introduction}

L'étude des mots infinis remonte au moins aux travaux de Thue ([13], [14]). Les régularités de ces mots ont dans un premier temps retenu l'attention ([13], [1], [7]) ; par la suite s'est posé le problème de la détermination du nombre $P(n)$ de facteurs distincts de longueur $n$. On a évidemment la majoration $P(n) \leq(\operatorname{Card}(A))^{n}$. Pour les mots infinis qui sont points fixes de substitutions uniformes sur un alphabet $A, \mathrm{~A}$. Cobham a montré [4] qu'il existe une constante $C$, dépendant du mot infini, telle que $P(n) \leq$ $C n$ et $\mathrm{N}$. Bleuzen-Guernalec [2] a précisé la constante en la majorant par $\sigma(\operatorname{Card}(A))^{2}$ ou $\sigma$ désigne le module de la substitution.

Nous construisons ici un automate pour la suite $n \rightarrow P(n+1)-P(n)$ lorsque le mot infini est minimal et point fixe d'une substitution injective uniforme sur un alphabet de cardinal deux.

\section{Préliminaires}

Soit $A^{*}$ le monoïde engendré par un ensemble fini non vide appelé alphabet. Les éléments de $A$ sont appelés lettres et ceux de $A^{*}$ mots. Pour tout mot $v$ de $A,|v|$ désigne la longueur de $v$, c'est-à-dire le nombre de ses lettres. L'élément neutre de $A^{*}$ est le mot vide noté $\varepsilon$. C'est le mot de longueur zéro. Soit $a \in A$; on note simplement $a^{*}$ pour l'ensemble $\{a\}^{*}$

Manuscrit reçu le 10 avril 1993, version définitive le 25 mars 1994. 
des mots finis formés avec la seule lettre $a$. Le mot infini formé avec la lettre $a$ est noté $a^{\infty}$.

Un mot $v$ est dit facteur de $w$ si $w=x v y$ avec $x$ et $y$ dans $A^{*}$. On écrit alors $v \mid w$. Si $x=\varepsilon$ (resp. $y=\varepsilon$ ), $v$ est dit préfixe (resp. suffixe) de $w$.

$p_{k}(w)$ (resp. $s_{k}(w)$ ) désigne le préfixe (resp. le suffixe) de $w$ de longueur $k$ $(\leq|w|)$. Un préfixe ou un suffixe de $w$ est dit strict s'il est différent de $w$.

On appelle substitution une application $f: A \rightarrow A^{*}$. Cette application se prolonge de manière naturelle en morphisme de monoïde $A^{*} \rightarrow A^{*}$. Une substitution $f$ est dite uniforme de module $\sigma$ si $\sigma=|f(i)|$ pour toute lettre $i$ de $A$, croissante si $|f(i)| \geq 2$. S'il existe une lettre $a$ de $A$ telle que $f(a)=a m$ avec $|m|>0$, alors l'ensemble des mots infinis de préfixe $a$ possède un point fixe $u=a m f(m) f^{2}(m) \cdots f^{k}(m) \cdots$. Une telle suite est automatique. On sait en effet ([5], [4]) que les suites projetées lettre à lettre de points fixes de substitutions de longueur constante sont exactement les suites engendrées par automates finis.

Soit $u$ un mot fini ou infini. L'ensemble des facteurs finis de $u$ est noté $F(u)$ et celui des facteurs de longueur $n$ est noté $F_{n}(u)$. Il est clair que tout facteur d'un mot $v$ de $F(u)$ est un mot de $F(u)$ et qu'il existe une lettre $a$ telle que $v a \in F(u)$. Le facteur $v$ de $u$ sera dit spécial si pour toute lettre $i$ de $A$, vi est facteur de $u$. $F S(u)$ désigne l'ensemble des facteurs spéciaux de $u$ et $F S_{n}(u)$ celui des facteurs spéciaux de longueur $n$.

Soit $S$ le décalage défini par $S\left(a_{0} a_{1} a_{2} \cdots\right)=a_{1} a_{2} \cdots$ et soit $\Omega$ l'adhérence de l'ensemble $\left\{S^{k}(u), k \in \mathbb{N}\right\}$ où la distance $d$ est définie par :

$$
d(v, w)=\exp \left(-\inf \left\{n \in \mathbb{N} ; v_{n} \neq w_{n}\right\}\right) .
$$

La suite $u$ est associé au système dynamique $(\Omega, T$ ), (où $T$ est la restriction de $S$ à $\Omega$ ), et est dite minimale si les seuls fermés invariants de $\Omega$ sous l'action de $S$ sont l'ensemble vide et $\Omega$.

La caractérisation suivante est classique [6] :

PROPOSITION 1. Le mot u est minimal si et seulement si pour tout facteur $m$ de $u$, il existe un entier $j$ dépendant de $m$ tel que :

$$
\forall k \in \mathbb{N}, m \mid u_{k} u_{k+1} \cdots \cdots u_{k+j} .
$$

Remarque 1. La condition (1) de la proposition 1 exprime que tout mot du langage de $u$ apparait dans $u$ avec des lacunes bornées (par $j$ ).

Lorsque $u$ est point fixe d'une substitution $f$, nous avons (voir [10]) un critère de minimalité en fonction de $f$ : 
Proposition 2. Soit u point fixe de la substitution $f$ sur l'alphabet $A$. Si a est préfixe de $u$ avec $|f(a)| \geq 2$ et si toutes les lettres de $A$ sont dans $u$, alors les propriétés suivantes sont équivalentes :

(i) $u$ est minimal et $\lim \left|f^{k}(b)\right|=+\infty$ pour toutes les lettres $b$ de $A$,

(ii) il existe $L \leq \operatorname{Card}(A)$ tel que pour tout $b \in A, a \mid f^{L}(b)$,

(iii) pour tout $b \in A$, il existe $k(b) \in \mathbb{N}$ tel que $a \mid f^{k(b)}(b)$.

Lorsque $A=\{1,2\}$, on a un critère de minimalité très simple :

Proposition 3. Soit $u$ un mot infini point fixe d'une substitution $f$ sur $A=\{1,2\}$ tel que 1 soit préfixe de $u$ et $u \neq 1^{\infty}$.

(i) Supposons $f$ croissante, alors : $u$ minimale $\Leftrightarrow f(2) \notin 2^{*}$.

(ii) Supposons $|f(1)| \geq 2$ et $f(2)=2$, alors : $u$ minimale $\Leftrightarrow f(1) \in$ $1 A^{*} 1$.

\section{Preuve}

(i) Si le mot est minimal, n'étant pas formé que de 1, il admet les facteurs $f^{k}(2)$. Donc si $f(2) \in 2^{*}$, le mot 1 apparait dans $u$ avec des lacunes arbitrairement grandes, contrairement à la proposition 1. Réciproquement si $f(2) \notin 2^{*}$, alors $1 \mid f(2)$ et la minimalité résulte de la proposition 2 .

(ii) Si 2 est suffixe de $f(1)$ alors on peut écrire $f(1)=1 B 12^{(b)}$ avec $b \geq 1$ et plus généralement $f^{k}(1)=1 B_{k} 12^{(k b)}$. Cette forme s'obtient par récurrence puisque $f^{k+1}(1)=\left(1 B 12^{(b)}\right) f\left(B_{k}\right)\left(1 B 12^{(b)}\right) 2^{(k b)}$. Ainsi les mots $2^{s}, s \geq 0$, sont-ils tous facteurs de $u$ qui n'est donc pas minimale. Supposons maintenant que 1 soit suffixe de $f(1)$. Posons $r=|f(1)|$. Le mot $2^{(r-1)}$ n'est pas facteur de $f(1)$ et s'il n'est pas facteur d'un mot $m$, il n'est pas facteur du mot $f(m)$. Il en résulte que 1 apparaît dans $u$ avec des lacunes bornées par $r-1$. Par suite $f^{k}(1)$ apparaît aussi dans $u$ avec des lacunes bornées et donc $u$ est minimale.

Dans tout ce qui suit, $u$ désigne une suite minimale non périodique, point fixe d'une substitution injective uniforme de module $\sigma$ sur un alphabet $A$.

3. Quelques propriétés des facteurs et facteurs spéciaux

Soit $m$ un facteur de $u$. In peut se factoriser sous la forme :

$$
m=x f(v) y
$$

avec les conditions 
$x$ suffixe strict d'un mot $f\left(v_{1}\right), y$ préfixe strict d'un mot $f\left(v_{2}\right)$ et $v_{1} v v_{2}$ facteur de $u$.

Un facteur $m$ de $u$ est dit mot rythmé s'il n'existe qu'un seul triplet $(v, x, y)$ vérifiant les conditions (2) et (3).

Proposition 4. S'il existe un facteur $R$ de u rythmé et de longueur $\geq \sigma$, alors tout facteur de $u$ dont $R$ est un facteur est aussi un mot rythmé.

\section{Preuve}

Soit $R$ un facteur de $u$ rythmé et de longueur $\sigma$. Soit $m$ un facteur de $u$ tel que $R$ soit un facteur de $m$. La factorisation de $u$ en blocs de $\sigma$ lettres, c'est-à-dire $u=f\left(u_{0}\right) f\left(u_{1}\right) f\left(u_{2}\right) \cdots$ donne une factorisation de $R$ lu dans $m$. Celle-ci étant unique, elle détermine alors un préfixe $B$ de $R$, de longueur $|B|<\sigma$ et tel que si $R=u_{k} u_{k+1} \cdots u_{k+\sigma}$, alors $k+|B|$ est un multiple de $\sigma$. La position de $u_{k+|B|}$ dans $R$, et par suite dans $m$, fournit une factorisation de $m$ induite par celle de $u$, mais celle-ci ne dépend pas de la lecture de $m$ dans $u$. Soit $m=\alpha\left(m_{1}\right)\left(m_{2}\right) \ldots\left(m_{t}\right) \beta$ cette factorisation unique où les mots $m_{i}$ sont dans $f(A)$ et les mots $\alpha$ et $\beta$ ont des longueurs strictement inférieures à $\sigma$. Le caractère injectif de $f$ donne la factorisation rythmée de $m$.

Proposition 5. Tout suffixe d'un facteur spécial est spécial.

\section{Preuve}

Soit $w=x v$ un facteur spécial. Pour toute lettre $i$ de $A, x v i=w i$ est un facteur de $u$ et $v i$ est donc un facteur de $u$. Ainsi $v$ est-il spécial.

Le corollaire suivant est immédiat :

Corollaire. Si $F S_{p}(u)$ est vide, alors pour tout $n \geq p, F S_{n}(u)$ est vide.

Le résultat assez classique suivant, cité dans [11], a été démontré dans [12] :

Proposition 6. Il existe $L_{0}$ dépendant de $\sigma$ et de $\operatorname{Card}(A)$ tel que tout facteur $m$ de $u$ de longueur plus grande que $L_{0}$ soit un mot rythmé.

Nous supposons à présent $A=\{1,2\}$.

\section{Calcul automatique}

Posons $\alpha=|P|$ où $P$ est le plus grand préfixe commun à $f(1)$ et $f(2)$ et montrons ceci : 
LEMME. Soit $k$ le plus petit entier tel que $\sigma k+\alpha \geq n$. Alors les facteurs spéciaux de longueur $n>L_{0}$ sont les suffixes de longueur $(n-\alpha)$ des images des facteurs spéciaux de longueur $k$ auxquels on a concaténé à droite $P$.

\section{Preuve}

Montrons dans un premier temps que la construction ci-dessus donne des facteurs spéciaux : soit $v$ un facteur spécial de longueur $k$. Alors, pour toute lettre $i$ de $A$, vi est un facteur de $u$. Il en est de même pour son image qui est de longueur $\sigma k+\sigma$.

Le préfixe $f(v) P$ de longueur $\sigma k+\alpha$ de l'image $f(v i)$ est spécial par définition de $P$. Par la proposition 5, il en est de même pour tous ses suffixes.

Montrons à présent que tout facteur spécial de longueur $n>L_{0}$ peut être obtenu par la construction du lemme : soit $w$ un facteur spécial de longueur $n>L_{0}$. Par la proposition $6, w$ est rythmé : $w=x f(v) y$, où $x$ est un suffixe strict de l'image par $f$ d'une lettre $j$ et $y$ préfixe strict de l'image par $f$ d'une lettre $k$. Ainsi $w$ est-il un facteur de $f(j v k)$ avec $j v k$ facteur de $u$. Comme $w$ est spécial, wi est un facteur de $u$ pour toute lettre $i$ de $A$. Chacun des $\operatorname{Card}(A)$ facteurs yi est un préfixe d'exactement un $f(k)$, la correspondance étant bijective. On a donc $y=P$ et $j v$ spécial. Finalement $w=x f(v) P$ où $x f(v)$ est un suffixe de $f(j v)$ avec $j v$ spécial.

Remarque 2. Pour deux lettres distinctes $i$ et $j$, désignons par $P_{i, j}$ le plus grand préfixe commun à $f(i)$ et $f(j)$. Pour un alphabet $A$ de cardinal quelconque, si pour tout couple $(i, j)$ de lettres distinctes de $A$ on a $P_{i, j}=$ $P,(P$ étant le plus grand préfixe commun aux images de toutes les lettres $\operatorname{de} A$ ), alors le lemme ci-dessus reste vrai.

Soit $P(n)$ le nombre de facteurs disctincts de longueur $n$ et soit $q(n)=$ $P(n+1)-P(n)$. On a le résultat suivant :

THÉORÈme 1. Soit $f$ une substitution injective uniforme de module $\sigma$ sur $A=\{1,2\}$, admettant un point fixe u minimal non périodique. Alors la suite $n \rightarrow q(n)$ ne prend qu'un nombre fini de valeurs.

\section{Preuve}

On a $q(n)=\operatorname{Card}\left(F_{n+1}\right)-\operatorname{Card}\left(F_{n}\right)=\operatorname{Card}\left(F S_{n}\right)$. Soit $L_{0}$ la constante de la proposition 6 et soit $E=\left\{q(n) ; 1 \leq n \leq L_{0}\right\}$. Posons $\mu=|S|$ où $S$ est le plus grand suffixe commun à $f(1)$ et $f(2)$. Si l'entier $n$ est écrit sous la forme $n=\sigma k+\alpha+r,(1 \leq r \leq \sigma)$, on sait (lemme ci-dessus) que les facteurs spéciaux de longueur $n$ sont obtenus à partir des facteurs spéciaux de longueur $(k+1)$. 
Deux facteurs spéciaux distincts de longueur $(k+1)$ ayant le même suffixe de longueur $k$ donneront (par la construction du lemme) le même facteur spécial de longueur $n$ si $r \leq \mu$. Pour $r>\mu$, il y a autant de facteurs spéciaux de longueur $n$ que de facteurs spéciaux de longueur $(k+1)$. Il vient donc que :

$$
\begin{aligned}
& \operatorname{Card}\left(F S_{n}\right)=\sum_{i} \operatorname{Card}\left\{s_{r}(f(a)), a=p_{1}(m), m \in F S_{k+1}, s_{k}(m)=m_{i}\right\} \\
&\left(\leq \operatorname{Card}\left(F S_{k+1}\right)\right) .
\end{aligned}
$$

L'entier $q(n)$ est donc parfaitement déterminé dès que $q(k+1)$ et $r$ sont connus. $\mathrm{Si}(k+1)>L_{0}$, on lui aplique le lemme pour obtenir $q(k+1)$. Ainsi pour tout $n>L_{0}, q(n) \in E$.

Pour $n>L_{0}$, si $\Phi_{r}$ désigne l'application de $E$ dans $E$ qui à $q(n)$ fait correspondre $q(k+1)$ lorsque $n=\sigma k+\alpha+r,(1 \leq r \leq \sigma)$, et $[x]$ la partie entière du réel $x$, on a donc le résultat suivant :

THÉORÈmE 2. Pour tout entier $n>L_{0}$, il existe un $\sigma$-automate (dont on a un programme formel ci-dessous) qui donne la valeur de $q(n)$ à partir de la décomposition de $n$ sous la forme $n=\sigma k+\alpha+r,(1 \leq r \leq \sigma)$ :

$$
\begin{aligned}
& n:=n_{\text {initial }} \\
& \Phi:=\text { Application identité dans } E \\
& \text { Tant que } n>L_{0}, \text { faire } \\
& k \leftarrow\left\{\begin{array}{l}
(n-\alpha) \sigma^{-1} \text { si entier } \\
1+\left[(n-\alpha) \sigma^{-1}\right] \text { sinon }
\end{array}\right. \\
& r \leftarrow n-\sigma k+1 \\
& \Phi \leftarrow \Phi \circ \Phi_{r} \\
& n \leftarrow k
\end{aligned}
$$

Fin de faire

$$
q\left(n_{\text {initial }}\right):=\Phi(q(n)) \text {. }
$$

\section{Exemple}

Soit $f$ la substitution sur $\{1,2\}$ définie $\operatorname{par} f(1)=12$ et $f(2)=21$. $f$ est injective. Le point fixe de $f$ de préfixe 1 est un mot infini $u$ minimal et non périodique. C'est la célèbre suite de Morse [9]. On observe facilement que les seuls mots rythmés dans $u$ de longueur 3 sont 221, 122, 112, 211, les autres ne sont pas rythmés $(121,212)$ ou ne sont pas des facteurs $(222,111)$. Soit $m$ un mot de longueur 4. Supposons qu'il n'admette pas de facteur rythmé de longueur 3 . Alors 121 ou 212 sont les seuls facteurs possibles de 
longueur 3 de sorte que $m=1212$ ou $m=2121$. On vérifie directement que ces deux mots sont des facteurs de $u$.

Supposons $m=1212=1 f(2) 2$. Étant facteur de $u$, le mot $21 f(2) 21=$ $f(222)$ est aussi facteur de $u$ et ne provient que de l'image par $f$ de 222 . $\mathrm{Par}$ suite le mot 222 est lui aussi facteur de $u$, ce qui est faux. Ainsi 1212 est rythmé, de même que 2121. Les autres facteurs de longueur 4 contiennent nécessairement l'un des quatres mots rythmés de longueur 3 . Finalement on a montré le résultat suivant (dont une version légèrement différente de trouve dans [10]) :

Proposition 7. Dans le mot de Morse, tout facteur de longueur supérieure ou égale à quatre est rythmé et la valeur quatre est optimale.

Un dénombrement direct donne $q(1)=q(2)=2$ et $q(3)=4$; on a alors $E=\{2,4\}$. Notons que $f(1)$ et $f(2)$ n'ont ni suffixe commun ni préfixe commun. Ainsi pour $n>4, q(n)=\operatorname{Card}\left(F S_{n}\right)=\operatorname{Card}\left(F S_{k+1}\right)$ où $2 k+r=n$ et $r=1$ ou 2. Donc $\Phi_{1}=\Phi_{2}=$ Identité dans $E$. Pour tout $n \geq 4$, il existe un unique entier $k$ tel que $2^{k+1}<n \leq 2^{k+2}$. Si $2^{k+1}+2^{k}<n \leq 2^{k+2}$, nous avons alors $3<n 2^{-k} \leq 4$ donc $4=n 2^{-k}$ si $n 2^{-k}$ est entier et $4=\left[n 2^{-k}\right]+1$ sinon et, par suite, $q(n)=q(4)=$ 2. Si $2^{k+1}<n \leq 2^{k+1}+2^{k}$, nous avons maintenant $2<n 2^{-k} \leq 3$ et le raisonnement précédent donne $q(n)=q(3)=4$. En remarquant que $3.2^{-2}<1 \leq 4.2^{-2}, 3.2^{-1}<2 \leq 4.2^{-1}$ et $3.2^{0}<4 \leq 4.2^{0}$, on a en définitive $q(n)=2$ pour les naturels $n$ pour lesquels il existe un entier relatif $k$ tel que $3.2^{-k}<n \leq 4.2^{-k}$ et $q(n)=4$ sinon.

Remarque 3. Le calcul automatique ci-dessus permet de retrouver (très rapidement) la formule explicite pour l'énumération des facteurs de la suite de Thue-Morse obtenue par Brlek [3] et par A. De Luca et S. Varricchio [8].

Note : B. Mossé a obtenu récemment des résultats qui généralisent ceux que nous avons exposés ici.

\section{BIBLIOGRAPHIE}

[1] S. Arson, Démonstration de l'existence de suites asymétriques infinies, Mat. Sb. 44 (1937), 769-777.

[2] N. Bleuzen-Guernalec, Suites points fixes de transductions uniformes, C. R. Acad. Sci. Paris, Série I 300 (1985), 85-88.

[3] S. Brlek, Enumeration of factors in the Thue-Morse word, Discrete Applied Math. 24 (1989), 83-96. 
[4] G. Christol, T. Kamae, M. Mendès France et G. Rauzy, Suites algebriques, automates et substitutions, Bull. Soc. math. France 108 (1980), 401-419.

[5] A. Cobham, Uniform tag Sequences, Math. Systems Theory 6 (1972), 164-192.

[6] W. H. Gottschalk and G. A. Hedlund, Topological dynamics, Am. Math. Soc. Colloq. Publ. 36, Providence R. I. (1968).

[7] Lothaire, Combinatorics on words, Addison Wesley MA (1982), chapter 12.

[8] A. de Luca and S. Varricchio, Some combinatorial properties of the Thue-Morse sequence and a problem in semigroups, Theoret. Comput. Sci. 63 (1989), 333-348.

[9] M. Morse, Recurrent geadesic on a surface of regative curvate, Trans. Amer. Math. Soc. 22 (1921), 84-100.

[10] M. Queffélec, Contribution à l'étude spectrale de suites arithmétiques, Thèse d'État, Paris-Nord, (1984).

[11] G. Rauzy, Rotation sur les groupes, nombres algébriques et substitutions, Séminaire de Théorie des Nombres, Bordeaux, exposé 21 (1987-1988), 21-1-21-12.

[12] T. Tapsoba, Complexité de suites automatiques, Thèse de troisième cycle, Université Aix-Marseille II (1987).

[13] A. Thue, Über unendliche Zeichenreihen, Norske Vid. Skr. I. Math. Kl., Christiana 7 (1906), 1-22.

[14] A. Thue, Über die gegenseitige Lage gleicher Teile genvisser Zeichenreihen, Norske Vid. Selsk. Skr. I. Math. Nat. Kl., Christiana 1 (1912), 1-67.

Théodore Tapsoba

Département de Mathématiques

Faculté des Sciences et Techniques

Université de Ouagadougou

03 B. P. 7021 Ouagadougou 03

Burkina Faso 